\title{
Estudio prospectivo en España: la tecnología wearable en el ámbito empresarial. Posibilidades como herramienta de comunicación
}

\author{
Prospective study in Spain: \\ wearable technology in corporate sector. \\ Possibilities as a communication tool
}

\author{
Esther Simancas González \\ Docente del departamento de Marketing y Comunicación \\ (Universidad de Cádiz) \\ Noelia María Moreno Mateo-Sidrón \\ Graduada en Publicidad y Relaciones Públicas \\ (Universidad de Cádiz)
}

Fecha de recepción: 25 de abril de 2017

Fecha de revisión: 19 de junio de 2017

Fecha de publicación: 1 de julio de 2017

Para citar este artículo: Simancas González, E. y Moreno Mateo-Sidrón, N. M. (2017): Estudio prospectivo en España: la tecnología wearable en el ámbito empresarial. Posibilidades como herramienta de comunicación, Icono 14, volumen 15 (2), pp. 220-243. doi: 10.7195/ ri14.v15i2.1083 


\section{MONOGRÁFICO}

\section{Resumen}

La presencia de los dispositivos wearable en el horizonte tecnológico plantea interrogantes aún no resueltos relacionados con su evolución y sus potencialidades en el campo de la comunicación y la publicidad. Considerando necesario prever y reflexionar acerca de estas transformaciones se ha realizado un panel Delphi, técnica de análisis prospectivo, conformado por los principales expertos en tecnología wearable en España. Los resultados de la investigación apuntan que esta tecnología está en una etapa inicial y que su éxito y penetración, tanto a nivel de usuarios como de empresas, dependerán de diferentes factores, como el valor añadido que sea capaz de aportar.

\section{Palabras clave}

Tecnología wearable - Internet de las cosas - Panel Delphi - Nuevas tendencias en comunicación - Nuevas tendencias en publicidad - Comunicación y tecnología.

\section{Abstract}

Wearable devices pose questions related to their development and their impact on the communication and advertising fields. A Delphi panel, as a prospective analysis technique, consisting of leading experts in the sector in Spain was carried out to reflect about possible effects. Results indicate that the wearable technology is still at an early stage and that its success and penetration will depend on different factors, such as value added to users and companies.

\section{Key Words}

Wearable technology - Internet of things - Delphi study - Communication trends Advertising trends - Communication \& technology. 


\section{Introducción}

La presente investigación trata de acercarse a la tecnología wearable para dilucidar cómo será su implementación como herramienta publicitaria y de comunicación por parte de las empresas. Ésta se postula como un estudio inicial, pues hasta ahora no se ha abordado este tema desde el ámbito científico ${ }^{1}$. Únicamente se han desarrollado investigaciones relacionadas con la tecnología wearable desde un plano más tecnológico o técnico; e incluso desde esta perspectiva, la mayoría de las aportaciones se centran en el internet de las cosas, abordando de forma muy superficial el estudio de los wearable. Basta con revisar las principales bases de datos científicas para comprobarlo.

Esta tecnología está cobrando cada vez más importancia (Fundación Telefónica, 2016) y podría marcar, como ha pasado con los smartphone, un antes y un después en la forma de interacción y relación entre las personas y con la propia tecnología, así como cambios a nivel empresarial. Por ello, hemos decidido realizar esta investigación de la mano de expertos profesionales en la materia para conocerla mejor, entender en qué etapa se encuentra y reflexionar acerca de su implementación en el marco empresarial como herramienta de comunicación.

Somos conscientes de las limitaciones y el esfuerzo prospectivo que supone realizar una primera aproximación a un objeto de estudio, que además está en pleno desarrollo, pero siguiendo a Salaverría (2015, p.223) se hace necesario "investigar en temas y áreas poco atendidos" para renovar la investigación y fomentar la innovación.

\subsection{Internet de la cosas}

El smartphone o teléfono móvil inteligente se está perfilando como una de las piezas claves en el acercamiento de los usuarios a los sistemas que permiten la interacción con objetos físicos (Thompson, 2005). En un período relativamente corto, su uso se ha generalizado y se emplea para realizar un gran número de tareas, algunas de las cuales necesitan de la interacción con objetos físicos de todo tipo para poder completarse. En este punto es donde puede hablarse de internet 
of things, traducido al español como internet de las cosas, considerado como la siguiente gran evolución del internet que se conoce hasta ahora, además de ser el origen a partir del cual se estructura la tecnología wearable, tema, este último, del que se ocupa la presente investigación.

Podría decirse que el origen del concepto de internet de las cosas surge en el Instituto Tecnológico de Massachusetts (MIT). Neil Gershenfeld, integrante del mismo, en su libro Cuando las cosas empiezan a pensar (1999) planteó la idea de que "el rápido crecimiento de la World Wide Web puede haber sido sólo la carga detonante para que las cosas empiecen a usar la red" (citado por Mattern y Floerkemeier, 2010, p.2). Pero sería Kevin Ashton, cofundador del Auto-ID Center (MIT), la primera persona en acuñar el término en una conferencia para Procter \& Gamble en 1999, en la que argumentó que, mediante la asociación de los objetos físicos con etiquetas $R F I D$, se podría dar identidad a cada objeto para generar datos sobre ellos (Haller, 2010; Kevin, 2009). Como señalan Alexandres Fernández, Rodríguez Morcillo García y Muñoz Frías (2006, p.46): “El objetivo de esta tecnología RFID (Radio Frequency IDentification) es el de identificar objetos de una forma rápida, con poca transmisión de información y en un entorno cercano".

Pero no será hasta 2005 cuando internet de las cosas alcance un mayor protagonismo con la publicación del primer informe sobre el tema por parte de la Unión Internacional de Telecomunicaciones (ITU). En éste se defendía que en un futuro esta tecnología conectaría objetos repartidos por todo el mundo, tanto de una forma sensorial como inteligente, mediante la combinación de los avances tecnológicos en la identificación de objetos, las redes de sensores, los sistemas integrados y la nanotecnología (Unión Internacional de Telecomunicaciones, 2005). Partiendo de esta idea, una definición más precisa de internet de las cosas sería

Infraestructura de red global dinámica con capacidad de auto configuración basada en protocolos de comunicación estándar e interoperables donde objetos físicos y virtuales poseen identidades, atributos físicos y personalidades virtuales, utilizan interfaces inteligentes y están perfectamente integrados en la red de información. (Clúster ICT - Audiovisual, 2013, p.15) 
Estudio prospectivo en España: la tecnología wearable en el ámbito empresarial... | 224

Podría decirse, por tanto, que internet de las cosas supone la evolución y la mejora de la computación ubicua y la inteligencia del entorno mediante una comunicación completa entre los objetos conectados a la red. En definitiva, y como ya hemos referido, podría considerarse como una prolongación del internet que se conoce hasta ahora, al permitir que pueda ser posible la conexión de "todas las cosas con todas las personas en una red mundial integrada" (Rifkin, 2014, p.24).

Torres Vargas y Arias Durá (2014) defienden que su desarrollo traerá consigo ventajas en el plano empresarial y económico, pues podrá contribuir a mejorar las experiencias y la satisfacción de los consumidores y usuarios, a aumentar la efectividad de costos o a optimizar la cadena de abastecimiento. No obstante, quedan cuestiones fundamentales por resolver. Uno de los mayores hándicaps es la vulnerabilidad de los usuarios en este entorno. Propuestas como la de Sánchez Alcón, López Santidrián y Martínez (2015) comienzan a aportar soluciones que garantizan la seguridad y privacidad de los datos personales.

\subsection{Tecnología wearable}

Dentro del mundo en pleno desarrollo de internet de las cosas, uno de los campos en los que se está poniendo mayor interés son los dispositivos wearable.

La wearable computing o tecnología wearable (también conocida como tecnología vestible) intenta desarrollar el concepto de usuario-máquina actuando como una sola identidad, siendo su fin último lograr desarrollar el hipotético concepto de cyborg, o simbiosis hombre-máquina, introducido por Clynes y Kline (1960). Este concepto se refiere a una combinación de la persona y la máquina, donde la máquina termina siendo una extensión natural del usuario que no requiere una atención consciente del mismo.

Este tipo de tecnología tiene como fin incorporarse en complementos o accesorios como relojes, pulseras, gafas, zapatillas e incluso ropa para convertirlas en objetos inteligentes que aporten una funcionalidad al usuario. Dicha funcionalidad puede partir desde la extracción de datos del propio usuario y su interacción con otros dispositivos, hasta la disposición de servicios externos. Cabe resaltar que la 
mayoría de dispositivos wearable creados hasta ahora compaginan su actividad con los smartphone, convirtiendo al móvil en el centro de la información de los usuarios, ya que el tamaño reducido de los dispositivos wearable no les permite tener una pantalla con el suficiente tamaño para ver y gestionar toda la información recogida por éstos.

Los wearable son dispositivos incorporados dentro del espacio personal del usuario con los que se produce una interacción constante (Rhodes, 2000). La idea principal es que el usuario pueda llevar siempre el dispositivo encima, aun cuando se encuentre en movimiento o realizando sus actividades cotidianas (Starner, 2001), permitiendo al usuario tener las manos libres.

Mann (1997) define los ocho atributos principales que ha de tener un dispositivo para considerarse wearable:

1. Constante: siempre estará activo aunque pueda tener algún modo de suspensión, a diferencia de un ordenador o portátil que debe encenderse antes de su uso.

2. Sin restricciones para el usuario: puede ser usado mientras el usuario hace cualquier otra actividad, es decir, no debe restringir la movilidad del usuario.

3. No monopolizan la atención del usuario: la utilización del dispositivo no te aísla del mundo exterior como ocurre, por ejemplo, con los juegos de realidad virtual. De hecho, intentará incluso mejorar las capacidades sensoriales del usuario.

4. Observables por el usuario: el usuario puede configurarlo para generar avisos o notificaciones.

5. Controlables por el usuario: el usuario puede tomar el control del dispositivo en el momento que desee, incluso en procesos automáticos. Por ejemplo, si se presiona accidentalmente un botón que implica una acción automática del dispositivo, el usuario puede detenerla inmediatamente. 
Estudio prospectivo en España: la tecnología wearable en el ámbito empresarial... | 226 MONOGRÁFICO

6. Atento al ambiente: es consciente del entorno, multimodal y multisensorial.

7. Comunicativo con otros: puede ser utilizado como un medio de expresión o como un medio de comunicación en cualquier momento que desee el usuario.

8. Personal: se convierte en una extensión del propio cuerpo del usuario. Es inseparable de él. Además, es privado, ya que sólo podría ser controlado por otra persona si el usuario poseedor del dispositivo se lo permitiera y autorizara.

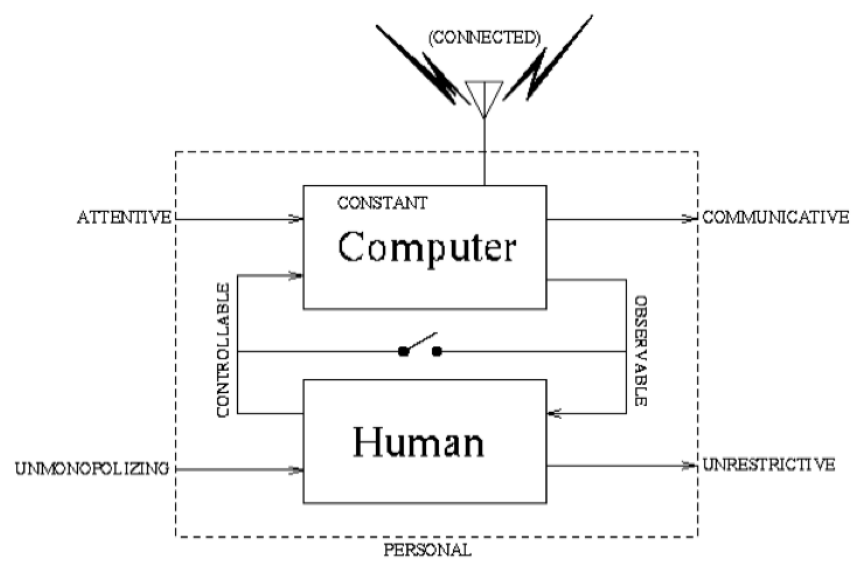

Figura 1: Atributos de la tecnología wearable.

Fuente: Mann (1997)

Según el informe de Fundación Telefónica (2016, p.81), “en el primer trimestre de 2016, se vendieron 19,7 millones de unidades" y "el fabricante que más unidades de wearable vendió fue Fitbit (4,8 millones), representando el $24,5 \%$ del mercado".

Existen diferentes áreas en las que se viene desarrollando esta tecnología, pero el ámbito de la salud es considerado uno de los pioneros en adaptarla de manera eficaz (Fundación Telefónica, 2016). Su aplicación en este campo se está llevando a cabo de diversas formas. Por un lado, se encuentran los dispositivos destinados al usuario 
pero que son controlados por un profesional médico, que adecua el aparato a las necesidades de salud del paciente; por otro lado, están los dispositivos wearable de uso personal y controlados por el propio usuario (categoría fitness, mayormente). Dentro de esta última categoría encontramos sobre todo pulseras inteligentes. Entre ellas, la que es considerada líder actual de mercado por marca es Fitbit.

Volviendo a aludir al informe de Fundación Telefónica (2016), más allá del sector de la salud, los wearable vienen consolidándose como una de las tendencias con más proyección en el mundo tecnológico. Castillo (2015) cree que esta tecnología capitaneará la nueva revolución tecnológica que está por venir.

De lo que no cabe duda es que el futuro de la comunicación empresarial se verá afectado por los cambios tecnológicos que se están produciendo (robotización, sistemas inteligentes, sistemas autónomos, internet of things, cloud, realidad virtual y wearable). Así ha sido hasta ahora: la comunicación corporativa y la publicidad han evolucionado de acuerdo a las transformaciones sociales y tecnológicas (Simancas y Bazaga, 2014).

¿Qué ocurrirá en el caso de los wearable? Se hace necesario comenzar a reflexionar desde el ámbito científico sobre sus posibles usos y consecuencias, más allá del plano meramente técnico.

\section{Material y método}

En este trabajo nos preguntamos por el estado actual y las tendencias de futuro de la tecnología wearable en España para:

- Ofrecer una visión general sobre la etapa en la que se encuentra la tecnología wearable en la actualidad.

- Conocer cómo será la adopción e incorporación de la tecnología wearable por parte de las empresas.

- Detectar posibles frenos y ventajas de la tecnología wearable para los usuarios. 
Estudio prospectivo en España: la tecnología wearable en el ámbito empresarial... | 228

- Investigar cómo podrá aprovecharse la tecnología wearable en las empresas como herramienta de comunicación, tanto a nivel interno como externo, y como plataforma publicitaria.

El estado de la investigación sobre la tecnología wearable se encuentra en una etapa embrionaria, por lo que para alcanzar los objetivos propuestos se optó por el empleo de la técnica Delphi, especialmente indicada para profundizar en temas complejos o realizar prospecciones acerca de cuestiones sobre las que hay poca información (Martínez Piñeiro, 2003). Tal como ocurre en el caso que presentan San Eugenio Vela, Fernández Cavia, Nogué y Jiménez Morales (2013, p. 660), “la necesidad de previsión de futuro y de fijación teórica y conceptual del objeto de estudio recomendaba su elección".

El método Delphi tiene como objeto la obtención de consenso sobre una materia concreta mediante el envío de cuestionarios secuenciales a expertos, sin que exista comunicación entre ellos para que no se produzcan injerencias (Uhl, 1991). Por lo tanto, la eficacia de la técnica y de sus resultados dependerá en gran medida de los expertos que participen en el panel. Para nuestro estudio se determinó que era fundamental que los panelistas contaran no solo con un alto grado de conocimiento en la materia, sino también con una fundada experiencia profesional en el área de la tecnología wearable, ya que en el ámbito científico, debido a la novedad del objeto de estudio, no existe aún una masa crítica de investigadores que haya abordado el estudio de los wearable desde el área de las ciencias sociales. Con todo, también se estableció como criterio básico para la elección de los especialistas que estos debían haber participado como ponentes en conferencias relevantes sobre el tema en España, como la Wearable Technology Conference o el IoT Solutions World Congress (ISWC) de Barcelona.

Tras contactar con veintitrés expertos, fueron diez los que finalmente formaron dicho panel, número que puede considerarse apropiado de acuerdo a autores como Landeta (1999).

El cuestionario inicial enviado a los panelistas estaba estructurado en tres bloques o módulos: 


\section{MONOGRÁFICO}

- Módulo I. Presente y futuro de la tecnología wearable: con preguntas generales sobre el estado actual y las previsiones de futuro de la tecnología wearable.

- Módulo II. La tecnología wearable en el ámbito empresarial: diseñado para prever cuestiones relacionadas con la adopción de esta tecnología por parte de las empresas y valorar su empleo como herramienta de comunicación.

- Módulo III. Usuarios: centrado en detectar posibles frenos y ventajas de la tecnología wearable para los usuarios, y conocer las posibilidades de esta como canal publicitario.

El cuestionario enviado contenía un total de 25 ítems, a evaluar por los expertos según su grado de acuerdo o desacuerdo, en una escala de 1 a 5 (escala Likert) ${ }^{2}$. Además, se ofrecía la posibilidad de que se pudieran añadir comentarios.

Para el procesamiento de los datos, con el programa informático SPSS se obtuvo una serie de parámetros estadísticos de las respuestas, a partir de los cuales se pudo determinar la homogeneidad o dispersión de las mismas, habiéndose fijado de antemano cuándo se produciría el consenso.

Con el objetivo de alcanzar el máximo consenso entre los expertos fueron necesarias dos rondas. La encuesta se envío por primera vez a todos los expertos en octubre de 2015 y una vez se procesaron los resultados, en pos de un mayor consenso, se volvieron a enviar las cuestiones no consensuadas adjuntándose las frecuencias de las respuestas para que, si lo consideraban oportuno, replanteasen su posición $\mathrm{y}$, en todo caso, la justificasen. Tras esta segunda vuelta finalizó el proceso ${ }^{3}$, pues se consideró que el consenso no aumentaría más en posteriores consultas. Finalmente, el proceso de recogida de datos acabó en enero de 2016.

\section{Resultados}

En la primera ronda, el consenso se alcanzó en el $60 \%$ de las cuestiones. En la segunda vuelta, el consenso alcanzado fue mayor, un 76\%, como puede comprobarse en la Tabla 1: 
Estudio prospectivo en España: la tecnología wearable en el ámbito empresarial... | 230

MONOGRÁFICO

\begin{tabular}{|c|c|c|c|}
\hline $\mathbf{1}^{\mathbf{0}}$ Fase & $\mathbf{N}^{0}$. Preguntas & Consenso & Sin consenso \\
\hline Módulo I & 8 & 4 & 4 \\
\hline Módulo II & 10 & 5 & 5 \\
\hline Módulo III & 7 & 6 & 1 \\
\hline TOTAL & 25 & 15 & 10 \\
\hline 2 $^{\circ}$ Fase & $\mathbf{N}^{0}$. Preguntas & Consenso & Sin consenso \\
\hline Módulo I & 8 & 7 & 1 \\
\hline Módulo II & 10 & 6 & 4 \\
\hline Módulo III & 7 & 6 & 1 \\
\hline TOTAL & 25 & 19 & 6 \\
\hline
\end{tabular}

Tabla 1: Consensos alcanzados en la primera fase del Panel Delphi

Organizadas por módulos, se expondrán a continuación las cuestiones que se plantearon a los expertos junto a los resultados estadísticos de las respuestas (media aritmética, mediana y desviación típica), obtenidos en la segunda y última vuelta del cuestionario. Asimismo, se analizarán de forma pormenorizada aquellos ítems en los que finalmente no se llega a consenso ${ }^{4}$, aportando las observaciones al respecto de los expertos que disienten.

Los resultados del primer módulo son los siguientes (Tabla 2):

\begin{tabular}{|c|c|c|c|c|}
\hline \multicolumn{2}{|c|}{ MÓDULO I } & MEDIA & MEDIANA & D.TÍPICA \\
\hline $\mathbf{1}$ & $\begin{array}{c}\text { Internet de las cosas y } \\
\text { la tecnología wearable } \\
\text { van a suponer una } \\
\text { nueva revolución digi- } \\
\text { tal en nuestra sociedad }\end{array}$ & 4,9 & 5,0 & 0,31623 \\
\hline $\mathbf{2}$ & $\begin{array}{c}\text { La penetración masiva } \\
\text { del smartphone ha sido } \\
\text { un paso primordial } \\
\text { para el surgimiento de } \\
\text { internet de las cosas y } \\
\text { la tecnología wearable }\end{array}$ & 4,8 & 5,0 & 0,42164 \\
\hline
\end{tabular}




\section{MONOGRÁFICO}

\begin{tabular}{|c|c|c|c|c|}
\hline \multicolumn{2}{|c|}{ MÓDULO I } & MEDIA & MEDIANA & D.TÍPICA \\
\hline 3 & $\begin{array}{l}\text { Tanto internet de las } \\
\text { cosas como la tecno- } \\
\text { logía wearable aún } \\
\text { se encuentran en una } \\
\text { etapa inicial }\end{array}$ & 4,6 & 5,0 & 0,51640 \\
\hline 4 & $\begin{array}{l}\text { La utilización del big } \\
\text { data será un factor } \\
\text { esencial para gestionar } \\
\text { la información gene- } \\
\text { rada por los objetos } \\
\text { conectados }\end{array}$ & 4,8 & 5,0 & 0,42164 \\
\hline 5 & $\begin{array}{c}\text { Con la introducción } \\
\text { de los wearable será } \\
\text { necesario proporcionar } \\
\text { unos servicios de se- } \\
\text { guridad y garantías de } \\
\text { privacidad mucho más } \\
\text { potentes que las que } \\
\text { rigen los smartphone, } \\
\text { tablet o } P C \text { actual- } \\
\text { mente }\end{array}$ & 4,3 & 4,5 & 0,82327 \\
\hline 6 & $\begin{array}{l}\text { La introducción de } \\
\text { Apple Watch o de Google } \\
\text { Glass en el mercado ha } \\
\text { sido determinante para } \\
\text { la expansión de los } \\
\text { dispositivos wearable }\end{array}$ & 3,1 & 3,0 & 0,99443 \\
\hline 7 & $\begin{array}{l}\text { Actualmente las po- } \\
\text { sibilidades de inde- } \\
\text { pendencia entre los } \\
\text { dispositivos wearable } \\
\text { y los smartphone son } \\
\text { poco probables }\end{array}$ & 2,2 & 2,0 & 1,03280 \\
\hline 8 & $\begin{array}{l}\text { Una vez que el nuevo } \\
\text { mercado de los dispo- } \\
\text { sitivos wearable llegue } \\
\text { a su fase de madurez, } \\
\text { estos llegarán a susti- } \\
\text { tuir al smartphone }\end{array}$ & 2,5 & 3,0 & 0,97183 \\
\hline
\end{tabular}

Tabla 2: Resultados estadísticos de las respuestas Módulo I 
Estudio prospectivo en España: la tecnología wearable en el ámbito empresarial... | 232

Dentro del primer módulo, la única cuestión a la que no se llegó al consenso fue la número 7. La frecuencia de las respuestas fue la siguiente: 2 en el valor 4 (de acuerdo), 6 en el valor 2 (en desacuerdo) y un 2 en el valor 1 (totalmente en desacuerdo). Los dos expertos que estaban de acuerdo con la afirmación y que por tanto disentían del resto justificaban su respuesta comentando que la mayoría de dispositivos wearable y su pantalla poseen un tamaño relativamente reducido. Esto se convierte en una barrera para que su funcionamiento pueda llegar a ser autónomo, ya que son utilizados como colectores de información pero dicha información posteriormente ha de verse reflejada en una pantalla.

Por otra parte, aunque en el ítem número 8 se llegó al consenso en la segunda fase, consideramos interesante exponer la aportación de un experto que disentía del resto al valorar con un 4 (de acuerdo) la afirmación. Justificó su decisión mediante la exposición de un ejemplo de prototipo en construcción desde comienzos de 2016 que podría cambiar la forma en la que conocemos y damos uso al smartphone hoy día: el Cicret Bracelet ${ }^{5}$. Es un brazalete que utiliza un picoproyector que proyecta en la piel del usuario, más concretamente en el antebrazo, la pantalla de su smartphone y éste podrá interactuar con ella, ya que incorpora ocho sensores de largo alcance que detectan las pulsaciones y gestos que se hagan sobre la piel. Este dispositivo cuenta con su propio procesador, conexión Wifi, Bluetooth y memoria externa. El usuario que lo utilice podrá realizar todas las funciones disponibles que permiten los sistemas operativos de los smartphone actuales, como conectarse a las redes sociales, mirar su bandeja de correo electrónico, activar la geolocalización o contestar y realizar llamadas, además de que es resistente al agua y se puede utilizar en cualquier lugar.

Pasando a los resultados del segundo módulo, pueden verse en la siguiente tabla (Tabla 3):

\begin{tabular}{|c|c|c|c|c|}
\hline \multicolumn{2}{|c|}{ MÓDULO II } & MEDIA & MEDIANA & D.TÍPICA \\
\hline $\mathbf{1}$ & $\begin{array}{c}\text { La evolución y desa- } \\
\text { rrollo de la tecnología } \\
\text { wearable promoverá el } \\
\text { surgimiento de nuevos } \\
\text { modelos de negocios } \\
\text { en las empresas }\end{array}$ & 4,1 & 4,0 & 0,73786 \\
\hline
\end{tabular}


233 | Esther Simancas González y Noelia María Moreno Mateo-Sidrón

\section{MONOGRÁFICO}

\begin{tabular}{|c|c|c|c|c|}
\hline \multicolumn{2}{|c|}{ MÓDULO II } & MEDIA & MEDIANA & D.TÍPICA \\
\hline 2 & $\begin{array}{l}\text { La adaptación de la } \\
\text { tecnología wearable } \\
\text { será posible para todo } \\
\text { tipo de empresas (na- } \\
\text { cionales, multinacio- } \\
\text { nales, pymes, etc.) }\end{array}$ & 4,1 & 4,5 & 0,99443 \\
\hline 3 & $\begin{array}{l}\text { La adopción de la } \\
\text { tecnología wearable } \\
\text { dentro de la empre- } \\
\text { sa implicará unos } \\
\text { elevados costes de } \\
\text { inversión }\end{array}$ & 2,7 & 2,5 & 0,82327 \\
\hline 4 & $\begin{array}{l}\text { La tecnología wearable } \\
\text { ofrecerá beneficios } \\
\text { a corto plazo a las } \\
\text { empresas que la imple- } \\
\text { menten }\end{array}$ & 3,2 & 3,5 & 1,03280 \\
\hline 5 & $\begin{array}{l}\text { El uso de la tecnología } \\
\text { wearable por parte de } \\
\text { una empresa conlle- } \\
\text { vará que se produzcan } \\
\text { cambios significati- } \\
\text { vos en el desarrollo } \\
\text { y organización de la } \\
\text { actividad de ésta }\end{array}$ & 3,3 & 3,5 & 0,94868 \\
\hline 6 & $\begin{array}{l}\text { Muchas empresas que } \\
\text { ya han implantado los } \\
\text { dispositivos wearable } \\
\text { dentro de sus procesos } \\
\text { de trabajo los utilizan } \\
\text { con el fin de controlar } \\
\text { a sus empleados }\end{array}$ & 2,0 & 1,5 & 1,15470 \\
\hline 7 & $\begin{array}{l}\text { Los dispositivos wea- } \\
\text { rable } \\
\text { mejorarán notable- } \\
\text { mente la productividad } \\
\text { y eficiencia de los } \\
\text { trabajadores de las } \\
\text { empresas }\end{array}$ & 3,7 & 4,0 & 1,05935 \\
\hline
\end{tabular}


Estudio prospectivo en España: la tecnología wearable en el ámbito empresarial... | 234

MONOGRÁFICO

\begin{tabular}{|c|c|c|c|c|}
\hline \multicolumn{2}{|c|}{ MÓDUL0 II } & MEDIA & MEDIANA & D.TÍPICA \\
\hline $\mathbf{8}$ & $\begin{array}{c}\text { Con la introducción de } \\
\text { la tecnología wea- } \\
\text { rable en la empresa, } \\
\text { habrá una mejora de la } \\
\text { comunicación entre los } \\
\text { distintos organismos o } \\
\text { departamentos que la } \\
\text { forman }\end{array}$ & 3,6 & 4,0 & 1,07497 \\
\hline $\mathbf{9}$ & $\begin{array}{c}\text { La utilización de dis- } \\
\text { positivos wearable en } \\
\text { las empresas aumen- } \\
\text { tará el sentimiento } \\
\text { de pertenencia de los } \\
\text { trabajadores }\end{array}$ & 3,1 & 3,0 & 0,56765 \\
\hline $\mathbf{1 0}$ & $\begin{array}{c}\text { Las empresas enfo- } \\
\text { carán la tecnología } \\
\text { wearable como una } \\
\text { herramienta de comu- } \\
\text { nicación externa con } \\
\text { sus clientes, más que } \\
\text { como una herramienta } \\
\text { de comunicación inter- } \\
\text { na con sus empleados }\end{array}$ & 3,6 & 3,0 & 0,84327 \\
\hline
\end{tabular}

Tabla 3: Resultados estadísticos de las respuestas Módulo II

En el segundo módulo, destinado a investigar posibles usos actuales y futuros de esta tecnología en el sector empresarial, es donde se ha producido un menor consenso, no habiéndose alcanzado en el $40 \%$ de las preguntas. Los panelistas sugirieron al respecto que las cuestiones de este módulo deberían haberse planteado de forma más concreta 0 segmentadas por sectores empresariales, pues la tecnología wearable tendrá diferentes aplicaciones según el tipo de empresa, su actividad o incluso su organización.

En relación al ítem número 4, hubo dos expertos que estaban en desacuerdo, alejándose de la media aritmética, y que mantuvieron sus respuestas en la segunda vuelta del cuestionario. Éstos explicaron que actualmente la tecnología wearable aún no ha llegado a un periodo de madurez, por lo que para que la implementación de esta tecnología en las empresas diera beneficios a corto plazo se necesita que ésta se estabilice y que tanto las empresas como sus trabajadores tengan amplios 


\section{MONOGRÁFICO}

conocimientos acerca de la misma, para que puedan aprovechar sus posibilidades y poder así rentabilizarla.

Estos argumentos serán los que esgriman nuevamente los expertos que discrepan con la idea: "los dispositivos wearable mejorarán notablemente la productividad y eficiencia de los trabajadores de las empresas" (cuestión número 7).

En la cuestión número 6, los expertos consideran, de acuerdo a los comentarios aportados, que las empresas que están implantando esta tecnología la utilizan con el fin de mejorar los procesos de trabajo, aunque no se descarta que pueda emplearse para ejercer un mayor control sobre los empleados, al ser computación altamente invasiva.

La última cuestión en la que no hay homogeneidad en las respuestas es la número 8. Los panelistas que discrepan exponen que la tecnología wearable podría mejorar la comunicación interna de las empresas, pero esto dependerá de la organización de la misma y de cómo se aproveche la tecnología. Añaden, además, que los smartphone ofrecen más posibilidades y facilidades de las que podría ofrecer un dispositivo wearable en este ámbito.

A continuación, se exponen los resultados del último módulo (Tabla 4):

\begin{tabular}{|c|c|c|c|c|}
\hline \multicolumn{2}{|c|}{ MÓDULO III } & MEDIA & MEDIANA & D.TÍPICA \\
\hline $\mathbf{1}$ & $\begin{array}{c}\text { El elevado precio de } \\
\text { este tipo de dispositi- } \\
\text { vos para los usuarios } \\
\text { supone una barrera } \\
\text { para la evolución y } \\
\text { expansión de la tecno- } \\
\text { logía wearable }\end{array}$ & 3,5 & 4,0 & 0,84984 \\
\hline $\mathbf{2}$ & $\begin{array}{c}\text { La creencia de la pér- } \\
\text { dida de la privacidad } \\
\text { es una gran barrera } \\
\text { para la incorporación } \\
\text { de los wearable en la } \\
\text { vida cotidiana de los } \\
\text { usuarios }\end{array}$ & 2,9 & 3,0 & 0,99443 \\
\hline
\end{tabular}


Estudio prospectivo en España: la tecnología wearable en el ámbito empresarial... | 236

MONOGRÁFICO

\begin{tabular}{|c|c|c|c|c|}
\hline \multicolumn{2}{|c|}{ MÓDULO III } & MEDIA & MEDIANA & D.TÍPICA \\
\hline 3 & $\begin{array}{l}\text { Uno de los objetivos } \\
\text { más importantes que } \\
\text { deberá cumplir un dis- } \\
\text { positivo wearable será } \\
\text { el de propiciar venta- } \\
\text { jas significativas a los } \\
\text { usuarios, ofreciéndole } \\
\text { herramientas útiles } \\
\text { para su vida diaria: re- } \\
\text { des de contacto, salud, } \\
\text { productividad, etc. }\end{array}$ & 4,6 & 5,0 & 0,51640 \\
\hline 4 & $\begin{array}{c}\text { A largo plazo, la tec- } \\
\text { nología wearable y sus } \\
\text { dispositivos se conver- } \\
\text { tirán en una prolonga- } \\
\text { ción del cuerpo del ser } \\
\text { humano, haciendo que } \\
\text { éste se olvide que lo } \\
\text { lleva puesto }\end{array}$ & 4,5 & 4,5 & 0,52705 \\
\hline 5 & $\begin{array}{c}\text { La publicidad en los } \\
\text { wearable provocará } \\
\text { una hiperpersonaliza- } \\
\text { ción de los mensajes } \\
\text { publicitarios }\end{array}$ & 4,5 & 5,0 & 0,70711 \\
\hline 6 & $\begin{array}{l}\text { La posible utilización } \\
\text { de los dispositivos } \\
\text { wearable como plata- } \\
\text { forma publicitaria será } \\
\text { sólo una extensión } \\
\text { de la publicidad y no } \\
\text { supondrá un cambio } \\
\text { significativo para el } \\
\text { mercado y la inversión } \\
\text { publicitaria }\end{array}$ & 1,7 & 1,0 & 1,05935 \\
\hline 7 & $\begin{array}{l}\text { La publicidad de las } \\
\text { marcas en los disposi- } \\
\text { tivos wearable puede } \\
\text { llegar a ser intrusiva } \\
\text { para los usuarios }\end{array}$ & 4,4 & 4,5 & 0,69921 \\
\hline
\end{tabular}

Tabla 4: Resultados estadísticos de las respuestas Módulo III 
Finalmente, en el tercer módulo se ha producido un desacuerdo en la cuestión número 6. En este caso, dos expertos frente al resto, que puntuaron el ítem con los valores 3 (neutral) y 4 (de acuerdo), creen que la utilización de los dispositivos wearable como plataforma publicitaria no supondrá en un principio un cambio significativo en el marco publicitario, pero habrá que tener en cuenta que al ser un dispositivo muy personal, la publicidad tendrá que estar limitada a momentos muy puntuales. Opinan que para que la tecnología wearable pueda convertirse en un nuevo área dentro del marketing tendrá que pasar mucho tiempo.

\section{Discusión de resultados}

De acuerdo a los resultados obtenidos, internet de las cosas y la tecnología wearable conforman la base tecnológica de lo que será una nueva revolución digital. Si consiguen tener un alto grado de éxito y de penetración, los dispositivos wearable podrían convertirse en una de las principales fuentes de información para internet de las cosas. También jugará un papel fundamental el big data pues, si los wearable están preparados para ser recolectores de información, este tendrá entre sus principales funciones almacenar, gestionar, transferir, analizar o visualizar la cantidad enorme de datos que recogerán los objetos conectados. Por tanto, los sistemas de gestión y procesado de datos tradicionales que se dedicaban a guardar y acumular la información se irán quedando poco a poco obsoletos, ya que no serán capaces de manejar, gestionar y analizar tal cantidad de información.

Los resultados muestran un claro acuerdo en que la tecnología wearable se encuentra actualmente en una etapa inicial. Esto explica la fuerte dependencia que tienen los wearable del smartphone, es decir, la necesidad de funcionar junto a él a la hora de visualizar datos o analizarlos. Esta dependencia se prevé que cambie cuando la tecnología vaya evolucionando, aunque para que pueda existir una total independencia se coincide en que habrá que buscar nuevas propuestas en relación al tamaño del dispositivo o de la pantalla de éste para que los propios datos que recoja pueda mostrarlos él mismo, sin necesidad de un smartphone u otro dispositivo complementario. Es importante destacar que, aunque los wearable consigan independizarse del smartphone, los expertos consideran que este último no será sustituido porque tanto los dispositivos wearable como los smartphone estarán enfocados para diferentes usos. Por otra parte, no cabe duda que la 
Estudio prospectivo en España: la tecnología wearable en el ámbito empresarial... | 238

alta penetración de los smartphone ha sido un aliciente para que empresas tecnológicas hayan decidido invertir en la creación y lanzamiento al mercado de estos dispositivos. Tanto es así que vemos cómo muchas de las grandes empresas tecnológicas, como Google, Android, Apple o Samsung, ya se han lanzado a crear y comercializar sus propios dispositivos wearable. También han aparecido nuevas empresas y start-ups cuyos productos son exclusivamente dispositivos wearable como Fitbit o FirstV1sion.

En cuestiones de seguridad y privacidad, los dispositivos wearable tienen mayor capacidad que los smartphone para recopilar información sobre los hábitos y preferencias de los usuarios. Así, podría afirmarse que la penetración masiva de los teléfonos inteligentes ha normalizado el uso de la tecnología de carácter intrusivo y esto ha favorecido que no se produzca un rechazo de los wearable, mucho más invasivos que los primeros. No obstante, teniendo en cuenta la información personal sobre comportamientos, hábitos o estado de salud de los usuarios que es capaz de registrar este tipo de dispositivos, los expertos consideran imprescindible que se ofrezcan garantías de seguridad capaces de crear un entorno seguro y fiable, para que los datos no puedan ser utilizados sin el consentimiento de los usuarios y la desconfianza no suponga una barrera en el desarrollo de esta tecnología.

El principal reto de la tecnología wearable es lograr una simbiosis entre la persona y el dispositivo. Se espera que este objetivo se cumpla a largo plazo y cuando esta tecnología alcance su etapa de madurez en el mercado, pero para ello será determinante que los dispositivos wearable sean capaces de aportar un verdadero valor añadido a quienes los adquieran, hecho que aún no se ha producido. Por ejemplo, uno de los expertos del panel Delphi aportaba que una de las razones de que el mercado de los smartwatch esté creciendo muy despacio es porque no aporta verdaderamente algo nuevo que no se pueda hacer ya con un smartphone. En este sentido, otro de los expertos comentaba que la expectación generada alrededor de las Google Glass y el Apple Watch es fruto principalmente de los esfuerzos comerciales de las compañías desarrolladoras más que de las ventajas competitivas que los productos en sí ofrecen. La cuestión es que este tipo de dispositivos ha de responder a las necesidades de los usuarios y todavía hoy no se está viendo un cambio significativo al respecto, aunque sí se está consiguiendo en otros campos del internet de las cosas, como es el caso del coche conectado. 
El precio en el mercado de estos dispositivos, sin embargo, no supondría un freno si se supera el hándicap que acabamos de comentar. Esto es, cuando esta tecnología se vaya consolidando y sea capaz de aportar beneficios y valor añadido a los usuarios, conseguirá tener éxito aunque su precio sea elevado.

Por otra parte, la mayoría de wearable lanzados al mercado están destinados a ser productos para el gran consumo, mientras que el enfoque de esta tecnología hacia la empresa aún no ha avanzado a gran escala en España. Se espera que esta situación cambie a medida que las empresas vayan conociendo las posibilidades que ofrecen los wearable para el almacenamiento, la monitorización, la gestión y el acceso a información desde cualquier lugar y en tiempo real. Lo que es seguro es que propiciará la creación y planteamiento de nuevos modelos de negocios, según apuntan los panelistas. Además, esta tecnología es considerada por los expertos como accesible a todo tipos de empresas (multinacionales, nacionales, pymes, etc.).

Una de las cuestiones que se planteaba en el cuestionario del panel Delphi era la utilización de los dispositivos wearable en la empresa con el fin de controlar a sus empleados. En este punto, los expertos estiman que las empresas que hasta ahora están incorporando el uso de los dispositivos wearable los están empleando como cuantificadores del trabajo de los empleados, no como instrumentos de control, aunque no descartan que puedan utilizarse para ejercer un mayor control sobre los empleados. En su mayoría, según explican, estos cuantificadores están centrados en la salud del trabajador. Dos expertos hacen referencia al programa de salud que Fitbit ha creado para las empresas llamado Corporate Wellness ${ }^{6}$. Explican que la monitorización del sueño, los pasos o la distancia recorrida diaria, al ser datos muy personales del usuario, sólo están accesibles para el administrador de manera general, no individual, es decir, sólo se puede observar la media de ese tipo de datos en un periodo concreto.

Cabría preguntarse, sin embargo, si el empleo de la tecnología wearable puede entenderse ajeno a la pérdida de intimidad de las personas, debate que se hace necesario y que ya viene produciéndose en relación al uso de internet y al big data.

En cuanto a la tecnología wearable como herramienta de comunicación empresarial, los expertos consideran que esta podrá aportar más o menos beneficios según el 
Estudio prospectivo en España: la tecnología wearable en el ámbito empresarial... | 240

tipo de compañía, sus objetivos, sus procesos de trabajo o su volumen de empleados. La empresa que desee implantarla podrá adquirirla y adaptarla a sus necesidades, pero su utilidad y eficiencia dependerán por encima de todo de que la empresa sea capaz de plantear y desarrollar una estrategia adecuada para el uso y aprovechamiento de la misma, partiendo para ello de un diagnóstico previo y siempre teniendo en cuenta la formación e implicación de los empleados. Por ello, con respecto a los costes de inversión, será fundamental que la empresa tenga en cuenta no sólo la adquisición de los dispositivos y la plataforma de gestión de éstos, sino también deberá destinarse un presupuesto a la formación de los empleados. De este modo, esta tecnología podría incidir positivamente en la mejora de la productividad y podría generar una gran conectividad en el seno de la empresa, siempre y cuando, señalan los panelistas, la tecnología sea empleada para favorecer a los empleados y los procesos de trabajo. Así, incluso repercutiría en un aumento del sentimiento de pertenencia.

En definitiva, la tecnología wearable podría convertirse en una herramienta de comunicación capaz de posibilitar mejoras en la comunicación interna de las empresas, fomentando tanto las relaciones de producción como las de identidad, pero no bastará únicamente con que se invierta en la adquisición de dispositivos y plataformas de gestión.

Un tema que se vuelve relevante en la evolución de la tecnología wearable es la influencia que esta podrá llegar a tener en el marco de la publicidad y el marketing. De acuerdo a los expertos, apoyándose en la información que podrá recabarse de los usuarios en el big data, esta tecnología incidirá tanto en la hiperpersonalización de los mensajes publicitarios como en la creación de nuevos formatos y soportes, lo que redundará en una mayor eficacia publicitaria. No obstante, desde nuestro punto de vista, esta nueva publicidad one-to-one sólo podrá contribuir a la relación marca-usuario si no tiene un carácter invasivo y es capaz de aprovechar creativamente las nuevas posibilidades que brinde el entorno.

Los panelistas creen que la gran oportunidad de la publicidad para con los wearable estará en los datos y en cómo se explote toda la información disponible. A nuestro modo de ver, la privacidad de los datos personales es precisamente el eje central sobre el que se debería reflexionar y aportar soluciones, pues, la tecnología wearable supone un paso más de lo que se ha vivido hasta ahora en cuanto a computación invasiva. 


\section{MONOGRÁFICO}

Por último, creemos que la investigación desarrollada y las conclusiones resultantes ofrecen una visión panorámica y bastante esclarecedora del estado actual de la tecnología wearable así como de sus potencialidades y limitaciones como herramienta de comunicación empresarial. Aunque también somos conscientes que al ser un estudio exploratorio, muestra una visión demasiado general $\mathrm{y}$, en pos de entender el funcionamiento y las características de la tecnología, mucho más técnica de lo que se pretendía en un principio. Sería interesante que las futuras investigaciones que aborden este objeto de estudio ahondaran en las posibles aplicaciones, usos y consecuencias sociales de esta tecnología como herramienta de comunicación.

\section{Notas}

[1] En el área de periodismo sí se acaba de publicar un estudio donde se analiza si los wearable son a día de hoy una opción interesante para la difusión de contenidos periodísticos (Silva Rodríguez, López García y Toural Bran, 2017).

[2] La elección de este formato de respuesta escalar responde a que este es el más utilizado en la técnica Delphi, al facilitar tanto la contestación del cuestionario como el análisis de los resultados (Landeta, 1999).

[3] Fernández - Ballesteros (1995) señala que el proceso puede finalizar cuando se prevea que el consenso no aumentará en posteriores consultas.

[4] Será en el siguiente apartado donde se discutan todos los resultados y se ofrezca una fotografía panorámica del tema que se aborda, tomando como base las puntuaciones y los comentarios y reflexiones de todos los panelistas.

[5] Veáse: http://cicret.com

[6] Véase: https://www.fitbit.com/es/fitbit-wellness

\section{Referencias}

Alexandres Fernández, S., Rodríguez Morcillo García, C. y Muñoz Frías, J.D. (2006). RFID: La tecnología de identificación por radiofrecuencia. En Anales de Mecánica y Electricidad, 83 (1), 47-52. Recuperado de goo.gl/rtTqXo

Ashton, K. (2009). That 'internet of things' thing. En RFiD Journal, 22 (7), 97-114. Recuperado de goo.gl/a0qUPw 
Estudio prospectivo en España: la tecnología wearable en el ámbito empresarial... | 242

Castillo, M. (2015). El IoT está cambiando los puestos de trabajo. En 2nd Wearable Technology and Internet of Things Conference. Instituto de la Economía Digital de ESIC. Recuperado de goo.gl/e3vDAR

Clynes, M.E. y Kline, N.S. (1960). Cyborgs and space. En Astronautics, september, 26-76. Recuperado de goo.gl/HWVt3t

Clúster ICT - Audiovisual (2013). Internet de las cosas: Objetos interconectados y dispositivos inteligentes. Recuperado de goo.gl/mFTBlE

De San Eugenio Vela, J., Fernández Cavia, J., Nogué, J. y Jiménez Morales, M. (2013). Características y funciones para marcas de lugar a partir de un método Delphi. En Revista Latina de Comunicación Social. doi: 10.4185/RLCS-2013-995 Fernández Ballesteros, R. (1995). Evaluación de programas: una guía práctica en ámbitos sociales, educativos y de salud. Madrid: Síntesis.

Fundación Telefónica (2016). La Sociedad de la Información en España 2015. Madrid: Ariel. Recuperado de goo.gl/j3PfyE

Haller, S. (2010). The things of internet of things. Poster at the Internet of Things Conference (IoT 2010), Tokyo. Recuperado de goo.gl/LwegcL

International Telecommunication Union (2005). The Internet of Things. ITU Internet Reports. Recuperado de goo.gl/RuJUqy

Landeta, J. (1999). El método Delphi, una técnica de previsión para la incertidumbre. Barcelona: Ariel.

Mann, S. (1997). Introduction: On the Bandwagon or Beyond Wearable Computing?. En Personal Technologies Journal, 1 (4), 203-207. doi: 10.1007/BF01682022

Martínez Piñeiro, E. (2003). La técnica Delphi como estrategia de consulta a los implicados en la evaluación de programas. En Revista de Investigación Educativa, 21 (2), 449-463. Recuperado de goo.gl/59IaoG

Mattern, F. y Floerkemeier, C. (2010). From the Internet of Computers to the Internet of Things. En K. Sachs, I. Petrov y P. Guerrero (Eds.), From Active Data Management to Event-Based Systems and More (pp. 242-259). Switzerland: Systems Group, Institute for Pervasive Computing, ETH Zurich. doi: 10.1007/9783-642-17226-7_15

Rifkin, J. (2014). La sociedad de coste marginal cero. El Internet de las cosas, el procomún colaborativo y el eclipse del capitalismo. Barcelona: Paidós Ibérica. Rhodes, B.J. (2000). Just-In-Time Information Retrieval agents. En IBM Systems Journal, 39 (3.4), 685 - 704. doi: 10.1147/sj.393.0685 


\section{MONOGRÁFICO}

Salaverría, R. (2015). Ideas para renovar la investigación sobre medios digitales. En El profesional de la información, 24 (3), 223-226. doi: 10.3145/epi.2015. may. 01

Sánchez Alcón, J.A, López Santidrián, L. y Martínez, J.F (2015). Solución para garantizar la privacidad en Internet de las Cosas. En El Profesional De La Información, 24 (1), 62-70. doi: 10.3145/epi.2015.ene.08

Silva Rodríguez, A., López García, X. y Toural-Bran, C. (2017). Los iWatch: el intenso flujo de microformatos de "periodismo de un vistazo" alimentan seis de los principales medios online. En Revista Latina de Comunicación Social, 72, 186-196. doi: 10.4185/RLCS-2017-1160

Simancas, E. y Bazaga, R. (2014). Nuevas tendencias en comunicación publicitaria. Red Bull y las Apps. En D. Caldevilla Domínguez (Coord.), Lenguajes y persuasión. Nuevas creaciones narrativas, 495-522. Madrid: ACCI.

Starner, T. (2001). The challenges of wearable computing: Part 1. En Ieee Micro, 21 (4), 44-52. doi: 10.1109/40.946681

Thompson, C.W. (2005). Smart devices and soft controllers. En IEEE Internet Computing, 9 (1), 82-85. doi: 10.1109/MIC.2005.22

Torres Vargas, G.A. y Arias Durá, R. (2014). El cómputo ubicuo y su importancia para la construcción del internet de las cosas y el big data.En Revista General de Información y Documentación, 24 (2), 217-232. doi: 10.5209/rev_RGID.2014. v24.n2.47401

Uhl, N.P. (1991). Delphi Technique. En A. Lewy (Ed.), The International Encyclopedia of curriculum (pp. 453-454). doi:10.4135/9781412958806.n266

Unión Internacional De Telecomunicaciones (1991). Recomendación X.800: Arquitectura de seguridad de la interconexión de sistemas abiertos para aplicaciones del CITT. Ginebra: Comité Consultivo Internacional Telegráfico y Telefónico. Recuperado de goo.gl/Rpo7xU 